\title{
Live Birth of a Healthy Boy after Preimplantation Genetic Testing for X-Linked Choroideremia Disorder
}

\author{
Tatsi P1*, Papoulidis $\mathrm{I}^{2}$, Timotheou $\mathrm{E}^{1}$, Chartomatsidou $\mathrm{T}^{1}$, Alexiou $\mathrm{M}^{2}$, Zafeiratis $\mathbf{0}^{1}$, Najdecki $\mathrm{R}^{1}$, Athanasiadis $\mathrm{A}^{3}$ and \\ Papanikolaou EG ${ }^{1,3}$ \\ ${ }^{1}$ Assisting Nature Unit, Centre of Reproduction and Genetics, Thessaloniki, Greece \\ ${ }^{2} A T G$ Access to Genome, Clinical Laboratory Genetics, Thessaloniki, Greece \\ ${ }^{3}$ Third Department of Obstetrics and Gynecology, Medical School, Aristotle University of Thessaloniki, Thessaloniki, Greece
}

\begin{abstract}
Preimplantation Genetic Testing (PGT) is a special technique in Assisted Reproduction Field that is applied to avoid a variety of hereditary disorders. This case report presents the first experience with rare X-linked Choroideremia disease (CHM), which leads to total blindness in male members of the family. To achieve a live birth in this case a total of 3 IVF procedures and 3 PGT cycles were performed. The data in our case offer the chance to couples suffering from rare genetic ophthalmo-neurological diseases to have healthy offspring.
\end{abstract}

Keywords: Preimplantation genetic testing $\bullet$ Choroideremia $\bullet$ Genetic diseases $\bullet$ X-linked $\bullet$ Assisted reproductive techniques

\section{Introduction}

Choroideremia $(\mathrm{CHM})$ is a rare $\mathrm{X}$-linked hereditary disorder that leads to the degeneration of the choriocapillaris, the retinal pigment epithelium, and the photoreceptor of the eye [1]. CHM causes progressive vision loss that finally leads to total blindness. It mainly affects males and the first symptom is usually nyctalopia at early childhood or puberty. Tunnel vision follows as the disease progresses and later the next symptom is visual acuity. The vision impairment worsens over time but the progression of choroideremia differs between patients, even within the same family [2]. Heterozygous females are mostly asymptomatic but often show striking funduscopic changes such as irregular pigmentation and atrophy around the optic disc [2-4]. Some fully affected females have been reported in the literature $[5,6]$.

Choroideremia is a rather rare disease since it affects almost 1 out of 50000-100000 people, but its frequency may be underestimated since there are similarities with other eye disorders that also lead to blindness. CHM is $X$-linked disorder that is inherited in a dominant pattern according to OMIM database (OMIM 303100). There is a $50 \%$ chance for the CHM gene to be passed from a parent onto an offspring.

Choroideremia is caused by loss of function in CHM gene that produces Rab escort Protein 1 (REP1) which attaches to Rab proteins in the cells and directs them to the lipid membrane of various organelles [7-9]. Rab escort play a significant role in intracellular trafficking and allow nutrients to pass across cells. In order to keep the biochemical pathways of vision operating while our eyes are open the above procedure is necessary. Mutations in the CHM gene lead either to an absence of REP-1 protein or the production of a protein that is not fully functional $[1-3,7,10-12]$. Premature cellular apoptosis is the result of the loss of function in REP-1 protein since the binding of Rab proteins to the organelle membranes is prevented.

*Address for Correspondence: Dr. Tatsi Petroula, Assisting Nature, Centre of Reproduction and Genetics, Thessaloniki, Greece, E-mail: tatsi@assistingnature.gr

Copyright: (c) 2020 Tatsi $P$, et al. This is an open-access article distributed under the terms of the Creative Commons Attribution License, which permits unrestricted use, distribution, and reproduction in any medium, provided the original author and source are credited.
There is no treatment for choroideremia and ultimately total blindness is caused to all patients. In this case, Preimplantation Genetic Testing (PGT) is an alternative method to prenatal diagnosis and its application prevents the transmission of the mutated gene that causes the disorder. Since its first application in 1990 PGT (Preimplantation Diagnosis) is available for innumerable rare genetic disorders. The aim of this paper is to show the application of Preimplantation Genetic Testing (PGT) for Choroideremia.

\section{Case Report}

\section{Family history}

The couple, a woman aged 32 and a man aged 36 , inquired genetic counseling for Choroideremia at our clinic due to male's family history. According to their family history, the husband's mother suffered from mere blindness and the older brother suffers from total blindness. His mother and brother attended an ophthalmology clinic and Choroideremia was suggested as a possible cause but unfortunately no genetic testing was carried out. Apparently, the CHM mutation is inherited to both brothers from their mother but due to the different progression patterns of the disorder even in the same family the husband does not yet experience all symptoms. He suffers from night blindness.

Before visiting our clinic, they were infertile for 40 months and the woman also suffered from endometriosis. After laparoscopic surgery, they tried to conceive with Intrauterine Insemination 3 times, all resulted negative. When they visited our clinic PGT was recommended after genetic testing of the husband for CHM mutation. The genetic testing came positive about $c .1125$ dup (p.Pro376Serfs*42). This mutation causes premature termination codon in the CHM gene and is probably the cause of Choroideremia. The whole PGT procedure was explained to the couple and they signed an informed consent (Figure 1).

\section{Mutation analysis}

Genomic DNA was extracted using the QIAamp DNA Blood Mini kit (Qiagen) according to the manufacturer's protocol. Multiplex PCR of the coding regions of the CHM gene (NM_000390.2; chr. X) and corresponding intron/exon boundaries ( $\pm 8 \mathrm{bp}$ ) was performed. Library preparation was performed by tagmentation (Nextera XT, Illumina), followed by next generation sequencing (MiSeq, Illumina). Alignment and variant calling were performed 


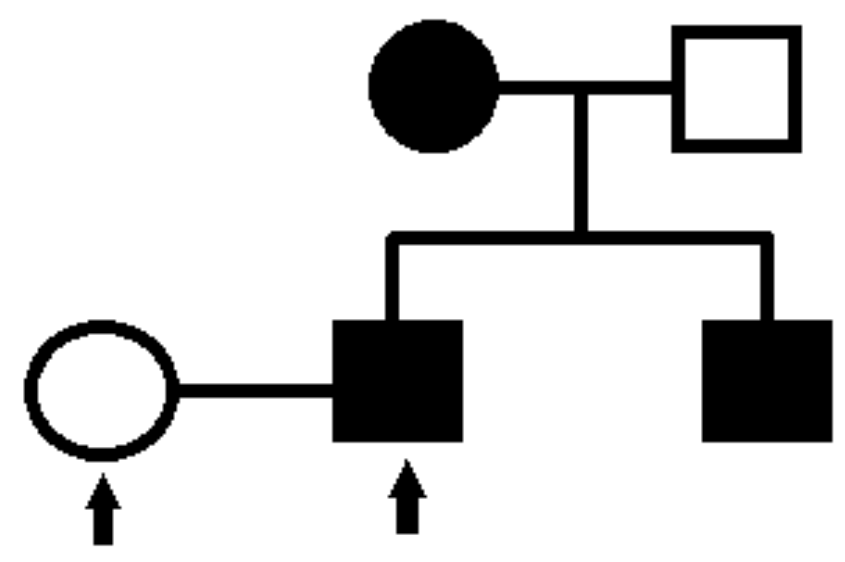

Figure 1. Family Tree showing the affected by CHM people in the family and the two arrows showing the couple in our case report.

using NextGENe software (SoftGenetics). Sanger sequencing was performed in order to confirm any detected variant [13-25].

\section{IVF Procedure 1 and $1^{\text {st }}$ PGT}

Regarding the IVF cycle, after ovarian stimulation using Long Protocol with gonadotrophin-releasing hormone agonist and recombinant follicle stimulant hormone for 10 days, 28 oocytes were retrieved. 18 oocytes were mature out of the 28 and 14 of them were normally fertilized (2pn) with ICSI. Finally, 12 embryos were vitrified on day-2 due to progesterone rise. Due to financial reason the couple decided to follow Preimplantation Genetic Testing for Aneuploidies (PGT-A) that also shows the sex of the embryo. In Greece sex selection is forbidden by the Law but is accepted in case of serious $\mathrm{X}$-linked genetic disorders.

Two months later, eight embryos were thawed on Day 2 and were biopsied the next day on Day 3. Cleavage Stage Biopsy technique was used. Vitrification and Thawing of the embryos on Day 2, is preferred due to the invasive nature of the biopsy technique. We prefer not to biopsy the embryos the same day as Thawing but to give them more time to relapse after Vitrification before Biopsy. One blastomere from each embryo was aspirated and sent for genetic testing. During the first PGT after array CGH analysis, the Preimplantation Genetic Testing for Aneuploidies showed four aneuploid embryos and four euploid embryos. Out of the normal embryos, two of them exhibit a normal male sex genomic pattern and were transferred on day 5 , blastocyst stage. After 10 days the hCG result was unfortunately negative.

\section{$2^{\text {nd }}$ PGT-A}

Two months later another Thawing and PGT-A cycle was performed and the analysis showed a chromosomally normal female embryo, an aneuploid embryo whereas the analysis of an embryo was not possible. So, the embryotransfer was cancelled and the couple was consulted to start a new ICSI cycle.

\section{IVF Procedure 2 and $1^{\text {st }}$ PGT-M}

After five months they decided to start a new ICSI/PGT cycle. Antagonist protocol was used for ovarian stimulation and on the day of the oocyte retrieval 16 oocytes were collected. 12 oocytes were fertilized with ICSI out of the 13 mature oocytes and 12 embryos were vitrified at the cleavage stage (day-2). On the Thawing cycle some months later, we decided to thaw only 6 embryos. Four embryos were suitable ( $>6$ cells) for Biopsy on day- 3 and four blastomeres were successfully removed. At this point PGT-M (Preimplantation Genetic Testing for Monogenetic Disease) was performed specifically for the mutation and not PGT-A/a-CGH. The preimplantation analysis showed three embryos where the variant (c.1125dup (p.Pro376Serfs*42)) was not detected. The analysis was not possible for one embryo.

Two embryos were transferred on day 5, while one was re-vitrified. Pregnancy test was positive and twin pregnancy was identified at 7 weeks.
Unfortunately, at twelve weeks vaginal bleeding was noticed and both embryos showed no heart activity. Induced abortion was performed and tissue from both sacs was sent for karyotype analysis. Only one sample was suitable for embryonic cells culture and that was detected with trisomy 18.

Some months later the normal for the mutation re-vitrified blastocyst was thawed and transferred but the result was negative [26-34].

\section{$3^{\text {rd }}$ PGT-A}

One month later the rest six embryos were thawed, four were suitable for biopsy and after PGT-A/CGH all four were aneuploid, and therefore no embryotransfer occurred. At this point they preferred PGT-A instead of PGT-M for financial reasons.

\section{IVF Procedure 3 and $4^{\text {th }}$ PGT-A}

Two months later they decided to start a new PGT cycle performing day 5 Biopsy. At this point we had just started practicing Trophectoderm Biopsy in our Lab. 21 oocytes were retrieved after Short Antagonist Protocol, 20 were fertilized and 4 of them vitrified on $2^{\text {nd }}$ culture day. The rest of them were cultured until Blastocyst stage. Trophectoderm Biopsy was performed in 6 of the embryos that reached Blastocyst Stage and were immediately vitrified one by one waiting for results. Sex selection was performed for six embryos at the blastocyst stage by using Polymerase Chain Reaction (PCR). Three of the embryos showed male sex genomic pattern. For these three male embryos Preimplantation Genetic Testing was performed and the analysis showed that one embryo was aneuploid whereas two embryos were euploid and their transfer is recommended.

On the next months a FRET cycle started and the two normal blastocysts were thawed and transferred. Ten days later the hCG test was positive. Preimplantation Genetic Testing (PGT-A) was performed by using two different analysis methods of aCGH to detect chromosomally normal embryos.

Whole Genome Amplification (WGA) was performed for each of the blastomeres or trophectoderm cells, using the SurePlex Single Cell Whole Genome Amplification kit (Bluegnome) or the PicoPlex ${ }^{\text {TM }}$ WGA kit (Agilent). All chromosomes (1-22, X, Y) were tasted for aneuploides (numerical

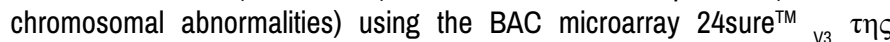
Illumina 2014 - formally BlueGnome or the microarray $8 \times 603 \mathrm{sl}$ GenetiSure Pre-Screen Array (Agilent Technologies). Data analysis was performed with the use of Blue Fuse Multi - Illumina Technologies or Cytogenomics software - Agilent Technologies.

Preimplantation Genetic Testing (PGT) analysis was performed, for the detection of the father's variant (c.1125dup (p.Pro376Serfs*42)), by amplifying the region of $\mathrm{CHM}$ gene containing the above variant using the PCR method (Polymerase Chain Reaction) and Sanger sequencing (Figure 2) [35-41].

\section{Results}

On the first ICSI cycle of this couple that PGT-A/aCGH was performed as mentioned the results proved that only 2 embryos were euploid boys and were transferred on day 5 , at the blastocyst stage, but the result was negative. In the next attempt not even one embryo was euploid so the embryotransfer was cancelled and a new ICSI cycle initiated that resulted in a pregnancy of twins that were later aborted. At this point the embryos were examined only for the CHM mutation with PGT-M after Day 3 biopsy. The embryos were not checked for aneuploidy and later when the embryos were tested after induced abortion due to no signs of heartbeat, the result showed trisomy 18 . This outcome and the PGT-A results from the previous cycle show clearly that there is also a high aneuploidy rate in the embryos retrieved from this couple. Consequently, the problem for this couple is not only Choroideremia but additionally high aneuploidy rate (Tables 1 and 2) [42-47].

\section{Discussion}

This is a report about c.1125dup (p.Pro376Serfs*42) mutation that causes 


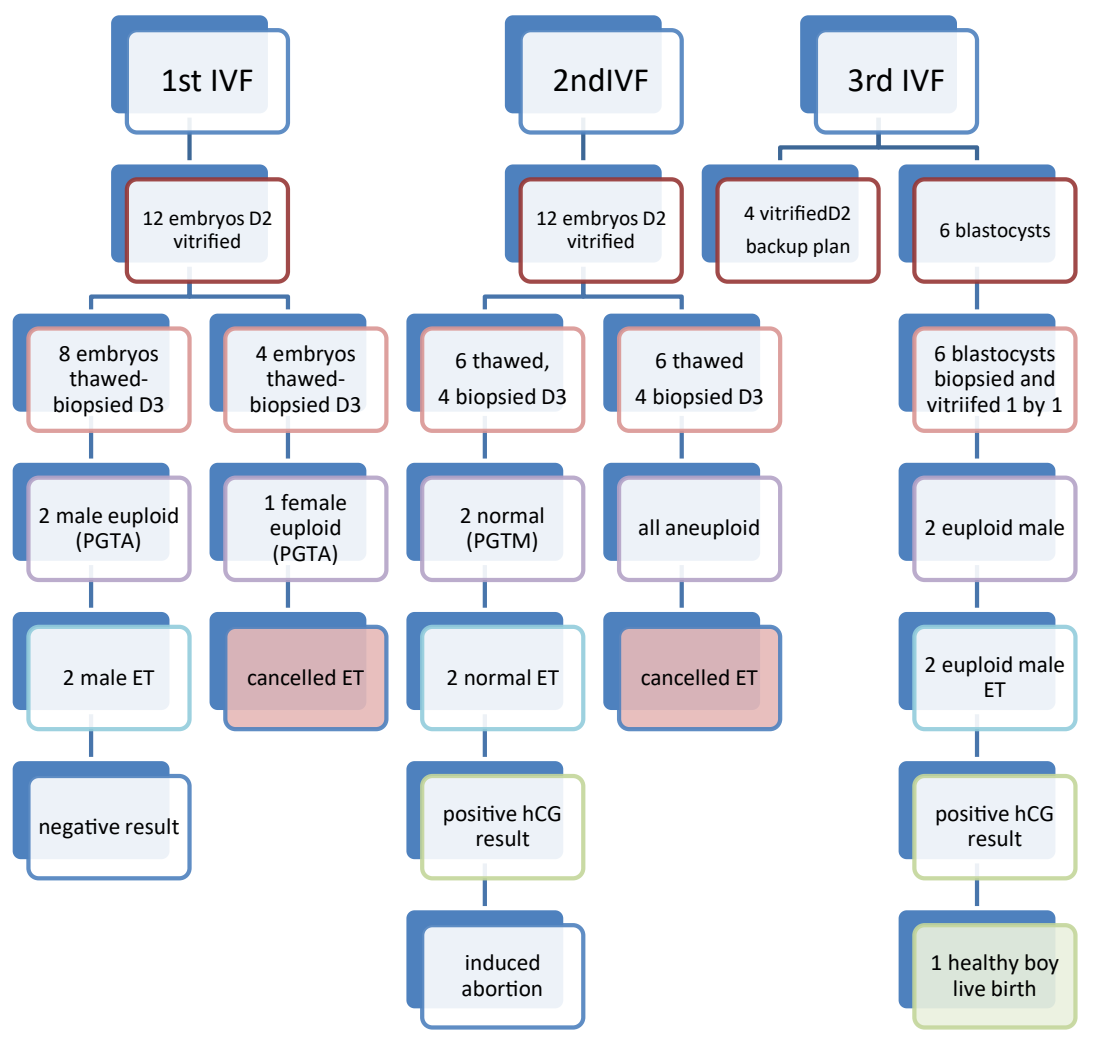

Figure 2. The road to a heathy live birth.

Table 1. 1st and 2nd IVF and D3 embryo biopsy- PGTA and PGTM.

\begin{tabular}{|c|c|c|c|c|c|c|c|c|c|}
\hline & cOCs & MII & $2 \mathrm{pn}$ & D2 embryos & $\begin{array}{l}\text { FRET-PGT } \\
\text { D3 biopsy }\end{array}$ & Biopsy Result & ET & Result & Live Birth \\
\hline \multirow[t]{2}{*}{ 1st IVF } & 28 & 18 & 14 & 12 Vitrified & 8 & 4 Euploid (2 Male, 2 Female) & 2 Male & Negative & No \\
\hline & & & & & 4 & 1 Euploid Female & Cancelled & Negative & No \\
\hline \multirow[t]{3}{*}{ 2nd IVF } & 16 & 13 & 12 & 12 Vitrified & $\begin{array}{l}6 \text { Thawed, } 4 \\
\text { Biopsied }\end{array}$ & $\begin{array}{c}3 \text { Normal } \\
\text { (2 Embryotransfer, } \\
1 \text { Revitrified) }\end{array}$ & 2 Normal & Positive & Abortion \\
\hline & & & & & & & 1 Normal Thawed & Negative & No \\
\hline & & & & & $\begin{array}{c}6 \text { Thawed, } 4 \\
\text { Biopsied }\end{array}$ & All Aneuploid & Cancelled & Negative & No \\
\hline
\end{tabular}

Table 2. 3rd IVF procedure and 4th PGT performing D5 biopsy.

\begin{tabular}{|c|c|c|c|c|c|c|c|c|c|}
\hline & cocs & MII & $2 \mathrm{pn}$ & D2 embryos & PGT-A D5 biopsy & Biopsy Result & FRET & Result & Live Birth \\
\hline $3^{\text {rd }}$ IVF & 21 & 21 & 20 & 4 Vitrified (Back-Up Plan) & 6 Blastocysts & 3 Males (2 Euploid, 1 Aneuploid) & 2 Male & Positive & 1 Boy \\
\hline
\end{tabular}

Choroideremia for which the husband came positive. This particular mutation causes a premature termination codon in the $\mathrm{CHM}$ gene as mentioned above.

A great deal of genetic diseases has been avoided since Preimplantation Genetic Testing was first implemented because only healthy embryos were selected for embryotransfer [48]. Nowadays, PGT for genetic testing is considered a routine procedure for every IVF clinic and an important alternative to prenatal diagnosis for several diseases such as beta-Thalassemia, Hemophilia A, Cystic Fibrosis, Duchenne's muscular dystrophy.

In our case specifically, the couple apart from being infertile was also carrier (the male) of Choroideremia, a rare genetic disease that causes blindness in boys. As mentioned above $\mathrm{CHM}$ is X-linked disorder. Therefore the couple was genetically counseled to have either Preimplantation Genetic Testing (PGT-M) for CHM (to exclude embryos being carriers of the disease) or Preimplantation Genetic Testing (PGT-A) with CGH array to test all 23 chromosomes for euploidy and thus to transfer only male embryos which would be unaffected for the mutation since the origin of the $\mathrm{X}$ chromosome was maternal.
In cases like this, where X-linked disease is to be avoided there are two options, either sex selection with PGT-A/a-CGH, or PGT-M specifically for the disease-causing mutation. The first approach ensures euploid embryos that do not carry or suffer the disease. The second approach that is more direct ensures an embryo that does not carry the mutated gene and consequently does not suffer from the disease. Unfortunately, PGT-M does not provide results regarding aneuploidy. From our experience with this couple that is also infertile and a high aneuploidy rate is detected, PGT-A/a-CGH for sex selection was the best option.

Before every PGT cycle pre-clinical testing and counselling work is necessary for the mutation to be detected, both partners should be tested for the best approach to be followed. In conclusion, a successful protocol for X-linked Choroideremia syndrome was developed, resulting in two clinical pregnancies. The first pregnancy of two healthy for the mutation-causing disease babies was terminated. Later a pregnancy with a healthy for $\mathrm{CHM}$ and euploid embryo followed, resulting in a live birth. In extremely rare conditions like this, a step by step strategy is the gold standard for couples at risk where the disease-causing mutation is known. 


\section{Conclusion}

In conlcusion, the biopsies that followed were tested with a-CGH not only for the specific mutation that causes CHM but also for euploidy while selecting healthy for the mutation-causing the disorder boys. After the last trial that a PGT-A/a-CGH was performed on day 5 embryos (Trophectoderm Biopsy) the two healthy embryos resulted in a healthy clinical pregnancy and live birth of healthy baby boy.

\section{Conflict of Interest}

None declared.

\section{References}

1. Cremers, F, D van de Pol, B Wieringa and F Collins, et al. "Molecular Characterization of Seven Microdeletions and Two X-Autosome Translocations in Patients with Choroideremia". Am J Hum Genet 45 (1989): A182.

2. Pameyer JK, PJ Waardenburg and HE Henkes. "Choroideremia". Brit J Ophthal 44 (1960): 724-738.

3. Bell, Alex G and JC McCulloch. Choroideremia and the Xg Locus: Another Look for Linkage. Clin Genet 2 (1971): 239-241.

4. Li, Shiqiang, Liping Guan, Shaohua Fang and Hui Jiang, et al. "Exome Sequencing Reveals CHM Mutations in Six Families with Atypical Choroideremia Initially Diagnosed as Retinitis Pigmentosa". Int J Molec Med 34 (2014): 573-577.

5. Gal, A, F Brunsmann, D Hogenkamp and $K$ Rüther, et al. "Choroideremia-Locus Maps Between DXS3 and DXS11 on Xq". Hum Genet 73 (1986): 123-126.

6. Shapiro I, and RJ Gorlin. "X-Linked Choroidal Sclerosis: A Stage of Choroideremia". Minnesota Med 57 (1974): 259-262.

7. Esposito, Gabriella, Francesca De Falco, Nadia Tinto and Francesco Testa, et al. "Comprehensive Mutation Analysis (20 Families) of the Choroideremia Gene Reveals a Missense Variant that Prevents the Binding of REP1 with Rab Geranylgeranyl Transferase". Hum Mutat 32 (2011): 1460-1469.

8. Handyside, Alan H, Elena H Kontogianni, KRML Hardy and Robert ML Winston. "Pregnancies from Biopsied Human Preimplantation Embryos Sexed by Y-Specific DNA Amplification". Nature 344 (1990): 768-770.

9. Seabra, Miguel C, Michael S Brown, Clive A Slaughter and Thomas C. Südhof. "Purification of Component A of Rab Geranylgeranyl Transferase: Possible Identity with the Choroideremia Gene Product". Cell 70 (1992): 1049-1057.

10. Cremers, FPM, F Brunsmann, TJR Van de Pol and IH Pawlowitzki, et al. "Deletion of the DXS165 Locus in Patients with Classical Choroideremia". Clin Genet 32 (1987): 421-423.

11. Cremers, Frans PM, Eeva-Marja Sankila, Frank Brunsmann and Marcelle Jay, et al. "Deletions in Patients with Classical Choroideremia Vary in Size from $45 \mathrm{~Kb}$ to Several Megabases". Am J Hum Genet 47 (1990): 622-628.

12. Wright, AF, RL Nussbaum, SS Bhattacharya and M Jay, et al. "Linkage Studies and Deletion Screening in Choroideremia". J Med Genet 27 (1990): 496-498.

13. Cheung, Marsha C, George $C$ Nune, Min Wang and Kerry E McTaggart, et al. "Detection of Localized Retinal Dysfunction in a Choroideremia Carrier". Am J Ophthal 137 (2004): 189-191.

14. Harris, Gordon $S$ and James $R$ Miller. "Choroideremia: Visual Defects in a Heterozygote". Arch Ophthal 80 (1968): 423-429.

15. Hodgson, SV, ME Robertson, CN Fear and J Goodship, et al. "Prenatal Diagnosis of X-Linked Choroideremia with Mental Retardation, Associated with a Cytologically Detectable X-Chromosome Deletion". Hum Genet 75 (1987): 286-290.

16. Jolly, Jasleen K, Markus Groppe, Jacqueline Birks and Susan M Downes, et al. "Functional Defects in Color Vision in Patients with Choroideremia". Am J Ophthal 160 (2015): 822-831.

17. Karna, Jussi. "Choroideremia: A Clinical and Genetic Study of 84 Finnish Patients and 126 Female Carriers". Acta Ophthal 176 (1986): 1-68.

18. Kurstjens, Joseph Hubert. "Choroideremia and Gyrate Atrophy of the Choroid and Retina". Doc Ophthal. 1965; 19: 1-122.
19. Lesko JG, RA Lewis, R Ferrell and RL Nussbaum. “Choroideremia is Tightly Linked to Two Proximal Xq Chromosomal Markers". Am J Hum Genet 37 (1985): A65.

20. MacDonald, Ian M, Dean Y Mah and YK Ho, et al. "A Practical Diagnostic Test for Choroideremia”. Ophthalmology 105 (1988): 1637-1640.

21. MacDonald, IM, RM Sandre, P Wong and AGW Hunter, et al. "Linkage Relationships of X-Linked Choroideremia to DXYS1 and DXS3". Hum Genet 77 (1987): 233-235.

22. Merry, Diane E, John G Lesko, Victoria Siu and Wayne F, et al. "DXS165 Detects a Translocation Breakpoint in a Woman with Choroideremia and a de novo X; 13 Translocation". Genomics 6 (1990): 609-615.

23. Mura, Marco, Christina Sereda, Monica M Jablonski and lan M MacDonald. "Clinical and Functional Findings in Choroideremia due to Complete Deletion of the CHM Gene". Arch Ophthal 125 (2007): 1107-1113.

24. Nussbaum, Robert L, John G Lesko, Richard Alan Lewis and Susan A Ledbetter et al. "Isolation of Anonymous DNA Sequences from within a Submicroscopic $X$ Chromosomal Deletion in a Patient with Choroideremia, Deafness, and Mental Retardation". Proc Nat Acad Sci 84 (1987): 6521-6525.

25. Nussbaum, RL, RA Lewis, JG Lesko and R Ferrell. "Choroideremia is Linked to the Restriction Fragment Length Polymorphism DXYS1 at Xq13-21". Am J Hum Genet 37 (1985): 473-481.

26. Ohba, Norio and Yasushi Isashiki. "Clinical and Genetic Features of Choroideremia". Jpn J Ophthal 44 (2000): 317.

27. Pascal O, P Donnelly, C Fouanon and O Herbert, et al. "A New (Old) Deletion in the Choroideremia Gene”. Hum Molec Genet 2 (1993): 1489.

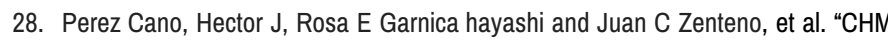
Gene Molecular Analysis and X-Chromosome Inactivation Pattern Determination in Two Families with Choroideremia". Am J Med Genet 149A (2009): 2134-2140.

29. Poloschek, Charlotte M, Barbara Kloeckener-Gruissem, Lutz L Hansen, Michael Bach, et al. "Syndromic Choroideremia: Sublocalization of Phenotypes Associated with Martin-Probst Deafness Mental Retardation Syndrome". Invest Vis Sci 49 (2008): 4096-4104.

30. Sankila, EM, A de La Chapelle, J Karna and H Forsius, et al. "Choroideremia: Close Linkage to DXYS1 And DXYS12 Demonstrated by Segregation Analysis and Historical-Genealogical Evidence". Clin Genet 31 (1987): 315-322.

31. Sankila, Eeva-Marja, Thomas Lehner, Aldur W Eriksson and Henrik Forsius, et al. "Haplotype and Multipoint Linkage Analysis in Finnish Choroideremia Families". Hum Genet 84 (1989): 66-70.

32. Sankila, Eeva-Marja, Ritva Tolvanen, Jose AJM van den Hurk and Frans PM Cremers, et al. "Aberrant Splicing of the CHM Gene is a Significant Cause of Choroideremia". Nature Genet 1 (1992): 109-113.

33. Schwartz, Marianne, T Rosenberg, E Niebuhr and C Lundsteen, et al. "Choroideremia: Further Evidence for Assignment of the Locus to Xq13-Xq21". Hum Genet 74 (1986): 449-452.

34. Schwartz, Marianne, Thomas Rosenberg, José AJM van den Hurk, Dorien JR van den Pol, et al. "Identification of Mutations in Danish Choroideremia Families". Hum Mutat 2 (1993): 43-47.

35. Schwartz, M, HM Yang, E Niebuhr and T Rosenberg. "Regional Localization of Polymorphic DNA Loci on the Proximal Long Arm of the $X$ chromosome using Deletions Associated with Choroideremia". Hum Genet 78 (1988): 156-160.

36. Seabra, Miguel C, Michael S Brown, and Joseph L Goldstein. "Retinal Degeneration in Choroideremia: Deficiency of Rab Geranylgeranyl Transferase". Science 259 (1993): 377-381.

37. Shapira, Theodore M and Jay A Sitney. "Choroideremia”. Am J Ophthal 26 (1943): 182-183.

38. Siu, Victoria M, John R Gonder, Jack $\mathrm{H}$ Jung and Frederick R Sergovich "Choroideremia Associated with an X-Autosomal Translocation". Hum Genet 84 (1990): 459-464.

39. Siu, Victoria M, John R Gonder, Jack H Jung and Frederick R Sergovich. "Choroideremia Associated with an X-Autosomal Translocation". (Abstract) Am J Hum Genet 43 (1988): A96.

40. Sorsby, Arnold, A Franceschetti, Ruby Joseph and JB Davey. "Choroideremia: Clinical and Genetic Aspects". Brit J Ophthal 36 (1952): 547-581.

41. Sorsby, Arnold and Mary Savory. "Choroidal Sclerosis: A Possible Intermediate Sex-Linked Form". Brit J Ophthal 40 (1956): 90-95. 
42. Syed, Nasreen, Julie E Smith, Sinoj K John and Miguel C Seabra. "Evaluation of Retinal Photoreceptors and Pigment Epithelium in a Female Carrier of Choroideremia". Ophthalmology 108 (2001): 711-720.

43. Tolmachova, Tanya, Ross Anders, Magnus Abrink and Laurence Bugeon, et al. "Independent Degeneration of Photoreceptors and Retinal Pigment Epithelium in Conditional Knockout Mouse Models of Choroideremia". J Clin Invest 116 (2006): 386-394.

44. Bokhoven, Hans van, Marianne Schwartz, Sten Andreasson and Jose AJM van den Hurk, et al. "Mutation Spectrum in the CHM Gene of Danish and Swedish Choroideremia Patients". Hum Molec Genet 3 (1994): 1047-1051.

45. van den, Hurk, José AJM, Dorien JR van de Pol and Bernd Wissinger, et al. "Novel Types of Mutation in the Choroideremia (CHM) Gene: A Full-Length L1 Insertion and an Intronic Mutation Activating a Cryptic Exon". Hum Genet 113 (2003): 268-275.

46. Van den Hurk, JAJM, TJR Van de Pol, CM Molloy and F Brunsmann, et al. "Detection and Characterization of Point Mutations in the Choroideremia Candidate Gene by PCR-SSCP Analysis and Direct DNA Sequencing". Am J Hum Genet 50 (1992): 1195-1202.

47. Preising, Markus and Carmen Ayuso. "Rab Escort Protein 1 (REP1) in Intracellular Traffic: A Functional and Pathophysiological Overview". Ophthalmic Genet 33 (2012): 57-65.

48. Handyside, Alan H, John G Lesko, Juan J Tarin and Robert ML Winston. "Birth of a Normal Girl After in vitro Fertilization and Preimplantation Diagnostic Testing for Cystic Fibrosis". N Engl J Med 327 (1992): 905-909.
How to cite this article: Tatsi P, Papoulidis I, Timotheou E, Chartomatsidou T, Alexiou M, Zafeiratis O, Najdecki R, Athanasiadis A and Papanikolaou EG. "Live Birth of a Healthy Boy after Preimplantation Genetic Testing for X-Linked Choroideremia Disorder." J Mol Genet Med 14 (2020): 450 doi: 10.37421/ jmgm.2020.14.450 